\title{
Disminución de la presión en un recipiente donde reacciona dióxido de carbono con una disolución básica
}

\author{
Antonio Tomás-Serrano ${ }^{1, a}$, Jerónimo Hurtado-Pérez ${ }^{2, b}$ \\ ${ }^{1}$ I.E.S. Vega del Táder. Molina de Segura (Murcia). España. \\ ${ }^{2}$ Museo de Ciencias MUDIC. Oribuela (Alicante). España. \\ atserrano@gmail.com,bjeronimob@gmail.com
}

[Recibido: 13 Febrero 2018. Revisado: 6 Mayo 2018. Aceptado: 29 Junio 2018]

Cuando en un recipiente cerrado, lleno en su mayor parte de gas, se lleva a cabo un proceso físico o una reacción química en la que disminuye la cantidad del gas presente, se originará una reducción de la presión que, en las condiciones adecuadas, puede dar lugar al aplastamiento del recipiente bajo la presión de la atmósfera. En este artículo se describe una forma sencilla de conseguir este llamativo resultado mediante una reacción química con materiales de fácil adquisición. Además, se indican algunas aplicaciones didácticas de este experimento, así como un método simple para estimar la disminución de presión que ocurre.

Palabras clave: Dióxido de carbono; Hidróxido sódico; Presión; Reducción de la presión; Fuerzas ejercidas por la atmósfera.

Decrease of pressure in a recipient where carbon dioxide reacts with a basic dissolution

When in a closed container, mostly filled with gas, a physical process or chemical reaction takes place producing a decrease of the amount of this gas, a pressure reduction will be caused, which, given the right conditions, can lead to the crushing of the container because of the pressure of the atmosphere. This article describes a simple procedure to achieve this remarkable result using a chemical reaction with materials that can easily be obtained. In addition, some educational applications of this experiment are provided, as well as a simple method to estimate the pressure drop that occurs.

Keywords: Carbon dioxide; Sodium hydroxide; Pressure; Pressure reduction; Forces exerted by the atmosphere.

Para citar este artículo: Tomás-Serrano A., Hurtado-Pérez J. (2018) Disminución de la presión en un recipiente donde reacciona dióxido de carbono con una disolución básica. Revista Eureka sobre Enseñanza y Divulgación de las Ciencias 15 (3), 3401. doi: 10.25267/Rev_Eureka_ensen_divulg_cienc.2018.v15.i3.3401

\section{Introducción}

Siempre con el objetivo de favorecer el interés de los estudiantes por la ciencia y facilitar así su aprendizaje, los autores de este artículo venimos trabajando desde hace mucho tiempo en el rediseño de prácticas convencionales de laboratorio para que se puedan desarrollar con los materiales presentes en cualquier laboratorio escolar e, incluso, con materiales caseros, como es el caso de la práctica que presentamos a continuación.

Uno de los experimentos más llamativos de física recreativa consiste en hacer un vacío parcial en el interior de un recipiente para que resulte aplastado por acción de la presión atmosférica. La forma más sencilla de crear este vacío es sustituir el aire por vapor de agua y provocar su condensación posterior, lo que acarreará una brusca reducción de la presión interna. En la figura 1 se muestra la secuencia de operaciones necesarias para conseguir este efecto con una lata de refresco (Shakhashiri 1985).

Otro método efectivo para reducir la presión interna de un recipiente, que está parcialmente ocupado por un gas, consiste en eliminar ese gas mediante una reacción química. El problema es que en la mayor parte de estas reacciones intervienen gases cuya obtención o manejo requiere la utilización de material convencional de laboratorio (como ocurre con dióxido de azufre, cloro, amoníaco, etc.) y no siempre se obtiene una reducción de presión tan grande y 
rápida como en el método anterior.

Un ejemplo de ello sería la reacción del oxígeno atmosférico con lana de hierro húmeda, para formar hidróxido de hierro (4 Fe(s) $+3 \mathrm{O}_{2}(\mathrm{~g})+6 \mathrm{H}_{2} \mathrm{O}(\mathrm{l}) \rightarrow 4 \mathrm{Fe}(\mathrm{OH})_{3}(\mathrm{~s})$ ), que aunque es muy sencilla de realizar (Prolongo-Sarria 2013), solo produce una disminución de la presión de alrededor de un $20 \%$ (esto es, el porcentaje en volumen de oxígeno en la atmósfera).
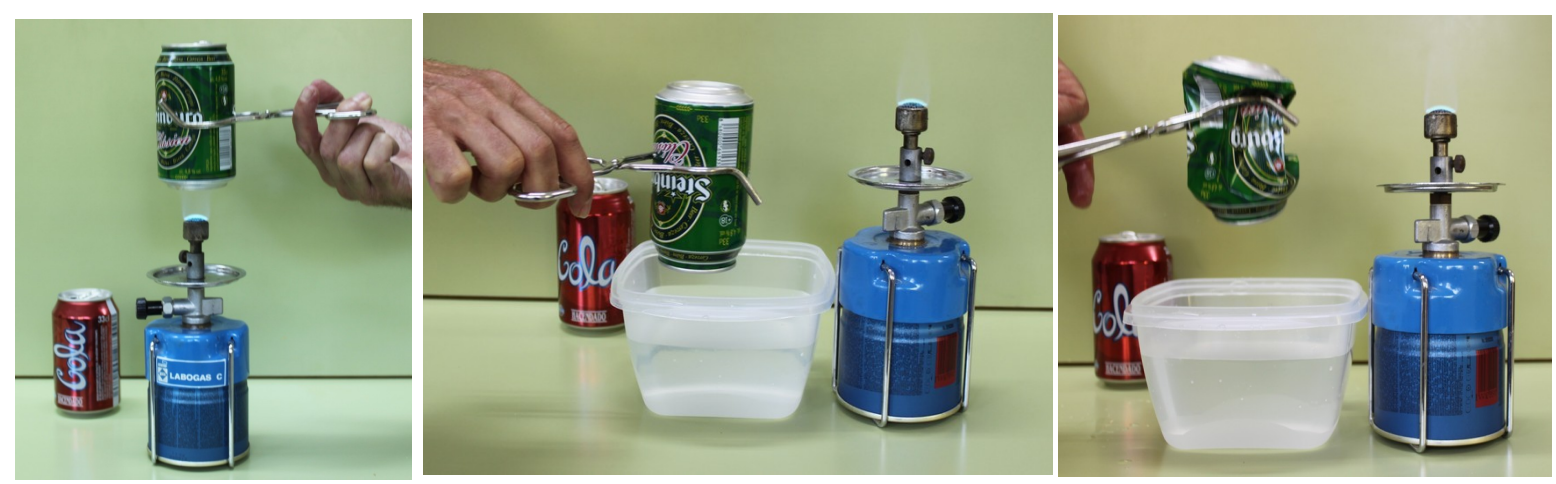

Figura 1. Se dispone una pequeña cantidad de agua en el interior de una lata de refresco y se calienta hasta que salga abundante vapor (izqda.). A continuación, se invierte la lata y se introduce rápidamente en un recipiente con agua fría (centro). Al condensarse el vapor de agua que llena la lata, la presión se hace prácticamente nula en el interior de la misma y resulta aplastada por la presión externa (dcha.).

En el experimento objeto de este artículo se salvan estos inconvenientes al utilizar la reacción de dióxido de carbono (un gas muy fácil de obtener y manejar) con una disolución de hidróxido sódico (IUB 2017).

\section{Reacción del dióxido de carbono con una disolución de hidróxido sódico}

El dióxido de carbono es un gas que se presta a numerosas experiencias escolares, tanto en el laboratorio como en casa. Una de las formas más sencillas de obtenerlo es hacer reaccionar hidrogenocarbonato de sodio, $\mathrm{NaHCO}_{3}$, con cualquier ácido como, por ejemplo, el ácido acético, $\mathrm{CH}_{3} \mathrm{COOH}$, o el ácido clorhídrico, $\mathrm{HCl}$ :

$$
\mathrm{NaHCO}_{3}(\mathrm{ac})+\mathrm{CH}_{3} \mathrm{COOH}(\mathrm{ac}) \rightarrow \mathrm{CO}_{2}(\mathrm{~g})+\mathrm{NaCH}_{3} \mathrm{COO}(\mathrm{ac})+\mathrm{H}_{2} \mathrm{O}(\mathrm{l})
$$

$\mathrm{Si}$ el $\mathrm{CO}_{2}$ obtenido reemplaza al aire de un recipiente y se hace reaccionar con una disolución de hidróxido sódico (o cualquier otra sustancia básica),

$$
\mathrm{CO}_{2}(\mathrm{~g})+2 \mathrm{NaOH}(\mathrm{ac}) \rightarrow \mathrm{Na}_{2} \mathrm{CO}_{3}(\mathrm{ac})+\mathrm{H}_{2} \mathrm{O}(\mathrm{l})
$$

ocurrirá una disminución de la presión interna del recipiente a medida que avance la reacción.

La diferencia de presión $\Delta p$ entre la presión externa (la presión atmosférica) y la interna del recipiente dará lugar a una fuerza neta $\Delta F$ sobre el recipiente, cuyo módulo vendrá dado por

$$
\Delta F=\Delta p \cdot S
$$

siendo $S$ la superficie de las paredes del recipiente (Resnick, Halliday y Krane 2002).

Como se mostrará más adelante, este procedimiento permite reducir la presión interna hasta en un $80 \%$ de su valor inicial, por lo que que la intensidad de la fuerza ejercida por la atmósfera es tal, que aplastará el recipiente si sus paredes no son lo suficientemente rígidas (véase la figura 2). 


\section{Materiales necesarios}

Las dos reacciones anteriores pueden llevarse a cabo con productos que encontramos fácilmente en nuestro entorno:

- El dióxido de carbono se puede preparar haciendo reaccionar vinagre ordinario (que, a efectos prácticos, se puede considerar como una disolución diluida de ácido acético) con bicarbonato de sodio de uso alimentario (hidrogenocarbonato de sodio).

- Se necesitará además hidróxido sódico (puede emplearse sosa comercial), una balanza y una probeta para medir las cantidades necesarias de los reactivos y una botella de plástico flexible de capacidad mayor o igual a $1 \mathrm{~L}$ (véase la figura 3); se obtienen buenos resultados con las botellas de gaseosa o de agua de 1,5 L de capacidad nominal (capacidad real de $1550 \mathrm{~cm}^{3}$ ).

\section{Planteamiento didáctico}

Este sencillo experimento admite varios enfoques en función del nivel de conocimientos del alumnado. Por ejemplo, se puede plantear como una demostración para ilustrar diferentes aspectos del estudio de los gases (presión, densidad, empuje hidrostático, fuerzas que ejerce la atmósfera, etc.) y de las reacciones químicas (estequiometría, variaciones energéticas, etc.). Pero también se puede enfocar como una experiencia de laboratorio o, incluso, como un trabajo de investigación dirigido a alumnos de bachillerato.

Veamos en primer lugar cómo utilizarlo para mostrar la intensidad de la fuerza que ejerce la atmósfera sobre los objetos que están en contacto con ella.
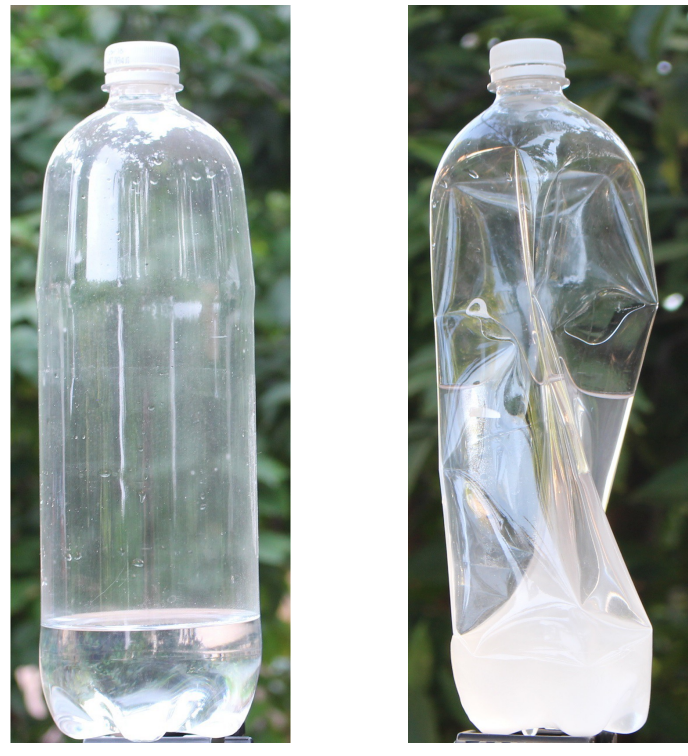

Figura 2. (Izqda.) Botella de plástico, de 1550 $\mathrm{cm}^{3}$ de capacidad real, con 1,4 $\mathrm{L}$ de dióxido de carbono, medido a $1 \mathrm{~atm}$ y $30{ }^{\circ} \mathrm{C}$, y $150 \mathrm{~cm}^{3}$ de disolución de hidróxido sódico, de concentración $25 \mathrm{~g} / \mathrm{L}$. (Dcha.) al reaccionar el dióxido de carbono con el hidróxido sódico, la presión interior disminuye y la botella colapsa bajo la fuerza que ejerce la atmósfera sobre sus paredes.

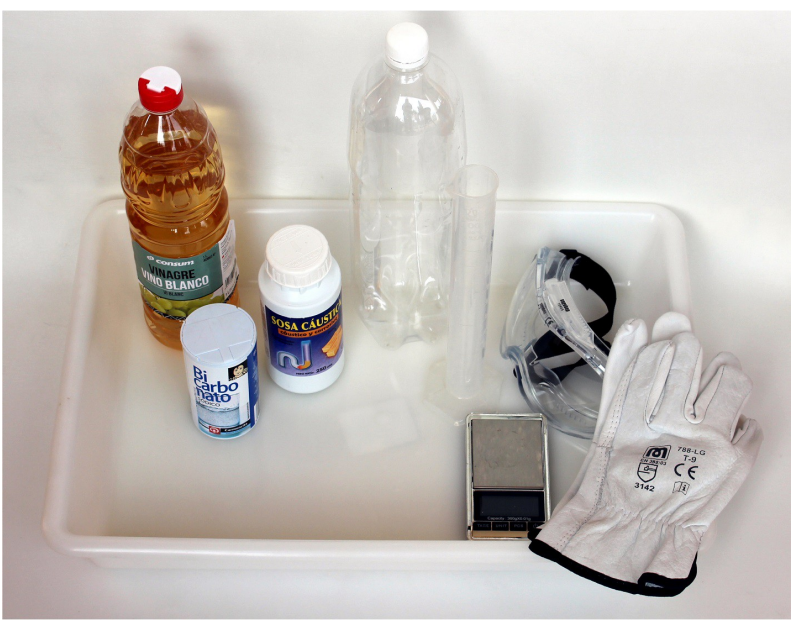

Figura 3. Materiales necesarios para realizar este experimento: de izquierda a derecha: vinagre de $6^{\circ}$ (disolución diluida de ácido acético), bicarbonato de uso alimentario (hidrogenocarbonato de sodio), sosa cáustica (hidróxido sódico), una botella vacía de gaseosa, probeta, balanza, gafas de seguridad y guantes. La misión de la cubeta de plástico es contener posibles derrames de las disoluciones utilizadas. 


\section{Colapso de una botella de plástico en cuyo interior disminuye la presión}

Se necesitará una botella de plástico flexible de 1,5 L, unos $100 \mathrm{~cm}^{3}$ de vinagre de $6^{\circ}, 8 \mathrm{~g}$ de bicarbonato de sodio y $7 \mathrm{~g}$ de escamas de hidróxido sódico (o cantidades proporcionales para recipientes de diferente capacidad).

Con estas cantidades de vinagre y bicarbonato de sodio (un $50 \%$ superiores a las estequiométricas) nos aseguraremos la producción de un exceso de dióxido de carbono que garantice el desplazamiento casi total del aire que contiene inicialmente la botella. La masa de hidróxido sódico también se ha incrementado en un $50 \%$ respecto a la cantidad estequiométrica para aumentar la velocidad de su reacción con el dióxido de carbono.

Si se preparan previamente las cantidades adecuadas de los reactivos, la demostración se realiza en unos minutos.

En caso de considerarlo oportuno, puede realizarse en primer lugar una prueba en blanco para comprobar que la reacción entre el ácido acético y el hidróxido sódico no ocasiona una disminución de la presión en el interior del recipiente. En realidad, la presión que ejerce el aire del interior de la botella aumentará ligeramente debido al incremento de temperatura que ocurre en este proceso.

Para llevar a cabo la demostración, se vierten el vinagre y el bicarbonato de sodio en la botella y se deja abierta, durante algo más de un minuto, para que el dióxido de carbono que se forma pueda desplazar y sustituir al aire que la llenaba inicialmente. Se puede confirmar que la botella está prácticamente llena de dióxido de carbono cuando se apaga una cerilla encendida al introducirla en la botella.

A continuación se agrega el hidróxido sódico, se enrosca firmemente el tapón y se agita repetidamente para favorecer el contacto del dióxido de carbono con la disolución de hidróxido sódico. En unos segundos, se apreciará que la botella comienza a aplastarse debido a la progresiva disminución de presión que ocurre en su interior (véase la figura 4).

Si se utiliza vinagre para generar el dióxido de carbono, se observará que la disolución cambia de color al añadir el hidróxido sódico. Esto se debe a que los polifenoles presentes en el vinagre actúan como un indicador ácido-base (Heredia-Avalos 2006). De hecho, si a la disolución final se le añade ácido clorhídrico, volverá a aparecer el color inicial del vinagre.

Aunque no es el objetivo principal de este trabajo, se puede utilizar un medidor de temperatura infrarrojo o, simplemente, introducir un termómetro ordinario en la botella (antes de colocarle el tapón) para observar las variaciones de temperatura que ocurren en estos procesos, ya que al formarse el dióxido de carbono, la temperatura desciende entre $3{ }^{\circ} \mathrm{C}$ y 4 ${ }^{\circ} \mathrm{C}$, pero al disolverse el hidróxido sódico y reaccionar con el dióxido de carbono, la temperatura de la disolución experimenta un aumento superior a los $10^{\circ} \mathrm{C}$.

$\mathrm{Si}$ al finalizar el experimento se quita el tapón, se escuchará el sonido que produce el aire al entrar a la botella (en cuyo interior la presión era menor que la atmosférica).

\section{Estimación de la presión en el interior del recipiente}

Una forma de estimar la disminución de presión que se genera con este método, consiste en medir el volumen de agua que absorbe un recipiente de paredes rígidas, una vez haya ocurrido la reacción entre el dióxido de carbono y el hidróxido sódico. Dado que la presión interior final puede llegar a ser muy baja (del orden de 0,1 atm), este experimento debe ser llevado a 
cabo por una persona experta; asimismo, se extremarán las precauciones para prevenir una posible, aunque poco probable, implosión del recipiente.

Se puede utilizar un matraz de vidrio con su correspondiente tapón para evitar, en la medida de lo posible, la entrada de aire al disminuir la presión interior (se puede mejorar el ajuste del tapón utilizando cinta de teflón). Las cantidades de los reactivos serán las correspondientes al volumen de este recipiente (para un matraz de 1 L se necesitarán unos $65 \mathrm{~cm}^{3}$ de vinagre de $6^{\circ}, 5 \mathrm{~g}$ de bicarbonato sódico y 4,5 g de hidróxido sódico).

Tras agregar el hidróxido sódico, se ajusta firmemente el tapón y se agita periódicamente, durante un par de minutos, para acelerar la reacción entre el dióxido de carbono y la disolución de hidróxido sódico. Acto seguido, se invierte el matraz, se introduce su cuello en una vasija con agua y se refrigera, vertiendo agua sobre él hasta que su temperatura sea aproximadamente igual a la temperatura ambiente. Finalmente, y manteniendo en todo momento la boca del matraz bajo el agua, se extrae el tapón (véase la figura 5).

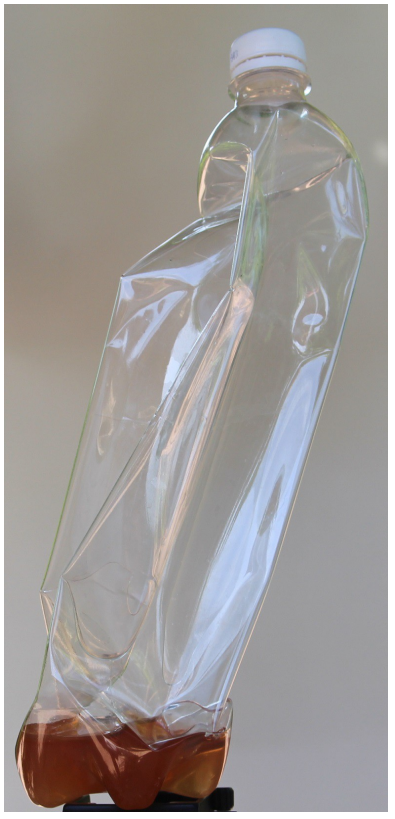

Figura 4. Estado final de la botella de plástico tras Como la presión interna $p_{1}$ es inferior a la atmosférica $p_{0}$, penetrará reaccionar el dióxido de agua al matraz hasta que la suma de la presión interna final $p_{2}$ y la presión que ejerce la columna de disolución de altura $b$ y densidad $d$, iguale a la presión atmosférica: carbono con el hidróxido sódico y resultar aplastada por la presión de la atmósfera.

$$
p_{2}+d \cdot g \cdot h=p_{\mathrm{o}},
$$

siendo $g$ la aceleración de la gravedad.

Si suponemos que los gases del interior del matraz (que son, fundamentalmente, aire, vapor de agua y dióxido de carbono) se comportan idealmente y tenemos en cuenta que la temperatura apenas varía en este proceso, podemos calcular el valor de la presión inicial $p_{1}$ utilizando la ley de Boyle

$$
p_{1} \cdot V_{1}=p_{2} \cdot V_{2},
$$

donde $V_{1}$ y $V_{2}$ son, respectivamente, los volúmenes de gas inicial y final. El volumen $V_{1}$ será aproximadamente igual a la diferencia entre la capacidad total del matraz y el volumen de vinagre utilizado, mientras que $V_{2}$ coincidirá con el volumen de agua necesario para acabar de llenar el matraz.

Como la altura de la columna de disolución es pequeña (aproximadamente, unos $25 \mathrm{~cm}$ ) y la densidad de esta disolución es solamente un $1 \%$ mayor que la del agua, se puede considerar que la presión final $p_{2}$ es prácticamente igual a la presión atmosférica $p_{0}$.

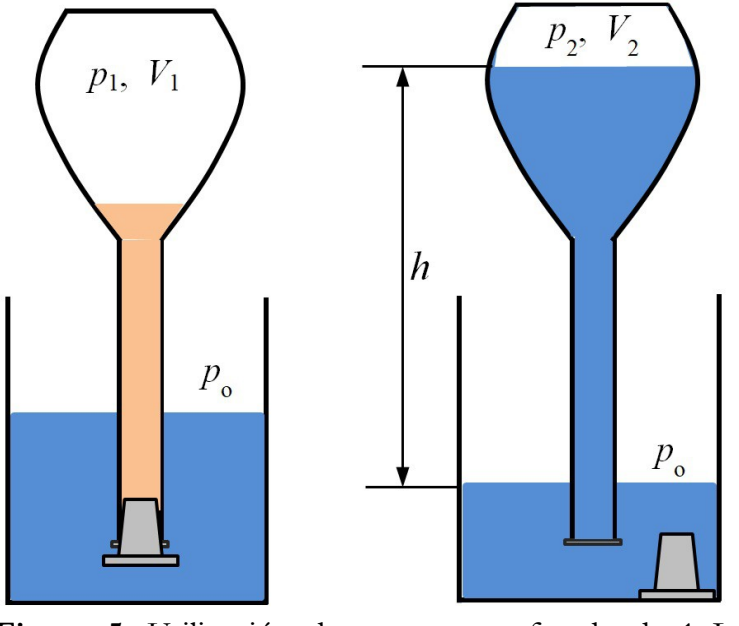

Figura 5. Utilización de un matraz aforado de $1 \mathrm{~L}$ Los resultados obtenidos en este experimento para estimar el valor de la presión $p_{1}$ tras la reacción
por los autores ponen de manifiesto que la del dióxido de carbono con la disolución de hidróxido presión interior $p_{1}$ es sensiblemente inferior a la sódico. atmosférica. 
Concretamente, con un matraz aforado de $1 \mathrm{~L}$ (de $1,020 \mathrm{~L}$ de capacidad real) y utilizando 65 $\mathrm{cm}^{3}$ de vinagre de $6^{\circ}, 5 \mathrm{~g}$ de bicarbonato sódico y $4,5 \mathrm{~g}$ de sosa cáustica comercial, se obtiene un valor medio para $V_{2}$ de, aproximadamente, $200 \mathrm{~cm}^{3}$ por lo que el valor obtenido para la presión interior $p_{1}$ en el matraz vale:

$$
p_{1}=\frac{p_{2} \cdot V_{2}}{V_{1}} \approx \frac{1 \cdot 0,2}{1,020-0,065}=0,21 \mathrm{~atm} .
$$

Esto es, se consigue una reducción de aproximadamente el $80 \%$ de la presión inicial.

Si se emplea un matraz de destilación o de filtración y se le acopla un tubito de silicona al tubo lateral, se facilitará la entrada de agua una vez que se haya reducido la presión dentro del recipiente.

En la práctica es difícil lograr que la presión $p_{1}$ sea menor de 0,2 atm por varias razones; en primer lugar, porque antes de que ocurra la reacción del $\mathrm{CO}_{2}$ con el hidróxido sódico en el interior de la botella, además de este gas, habrá una pequeña cantidad de vapor de agua y de aire; por otra parte, la citada reacción no transcurrirá completamente y, por último, si el tapón no ajusta perfectamente, permitirá la entrada de algo de aire al interior del recipiente cuando comience a disminuir la presión.

\section{Planteamiento como una práctica de laboratorio}

Se le puede dar un enfoque más académico a este experimento y plantearlo como una práctica de laboratorio, dirigida a estudiantes de bachillerato.

En primer lugar, como ejemplo práctico de cálculos estequiométricos, se les solicitaría a los estudiantes la realización de los cálculos para determinar las cantidad de ácido acético (a partir de vinagre de $6^{\circ}$ ) e hidrogenocarbonato de sodio necesarios para obtener el dióxido de carbono que se empleará en el experimento. Deberá tenerse en cuenta que se necesita producir un exceso de dióxido de carbono (como mínimo del 50\%) para garantizar que pueda reemplazar la máxima cantidad posible del aire contenido inicialmente en el recipiente en que se llevará a cabo el experimento.

A continuación se calculará la masa de hidróxido sódico necesaria para que reaccione totalmente el dióxido de carbono contenido en el recipiente. Será necesario incrementar la cantidad estequiométrica en un mínimo del $20 \%$ para que la reacción con el dióxido de carbono transcurra con mayor rapidez.

Si la práctica tiene como objetivo la simple observación de la disminución de la presión, la reacción se llevará a cabo en una botella de plástico flexible. Si se desea un enfoque cuantitativo y medir la variación de presión originada por esta reacción, entonces deberá utilizarse un matraz de vidrio de una capacidad mayor o igual a $0,5 \mathrm{~L}$, repitiendo los pasos y cálculos descritos en el apartado anterior.

\section{Reacción entre el dióxido de carbono y el hidróxido sódico en fase sólida}

Ya se ha mencionado anteriormente que, para que la reacción entre el dióxido de carbono y el hidróxido sódico ocurra rápidamente, hay que agitar repetidamente el recipiente para favorecer el contacto entre la fase gaseosa (dióxido de carbono) y la acuosa (disolución de hidróxido sódico). El uso de disoluciones y la agitación aumentan la velocidad de reacción (Brown, LeMay y Bursten 1998).

Para resaltar este hecho, se puede repetir el experimento, pero de modo que la reacción ocurra ahora entre una fase gaseosa $\left(\mathrm{CO}_{2}\right)$ y una fase sólida $(\mathrm{NaOH})$. 
Para ello habrá que producir el dióxido de carbono en otro recipiente y trasvasarlo a la botella en la que queremos que ocurra su reacción con el hidróxido sódico. En la figura 6 se muestra un esquema del dispositivo experimental que puede utilizarse.

La diferencia es que ahora la reacción es mucho más lenta y se consigue una menor disminución de la presión. No obstante, a medida que pasa el tiempo y el hidróxido sódico se transforma en carbonato de sodio, se aprecia perfectamente cómo se comprime la botella y cambia la textura de la fase sólida.

Para confirmar que, efectivamente, se ha formado carbonato de sodio, al finalizar el experimento se le pueden añadir unas gotas de una disolución ácida (p. e. vinagre) al sólido del interior de la botella y comprobar que se liberan burbujas de gas como resultado de una reacción característica de los carbonatos e hidrogenocarbonatos, $\mathrm{CO}_{3}{ }^{2-}+2 \mathrm{H}^{+} \rightarrow \mathrm{CO}_{2}+\mathrm{H}_{2} \mathrm{O}$ (Skoog, West y Holler 1997).
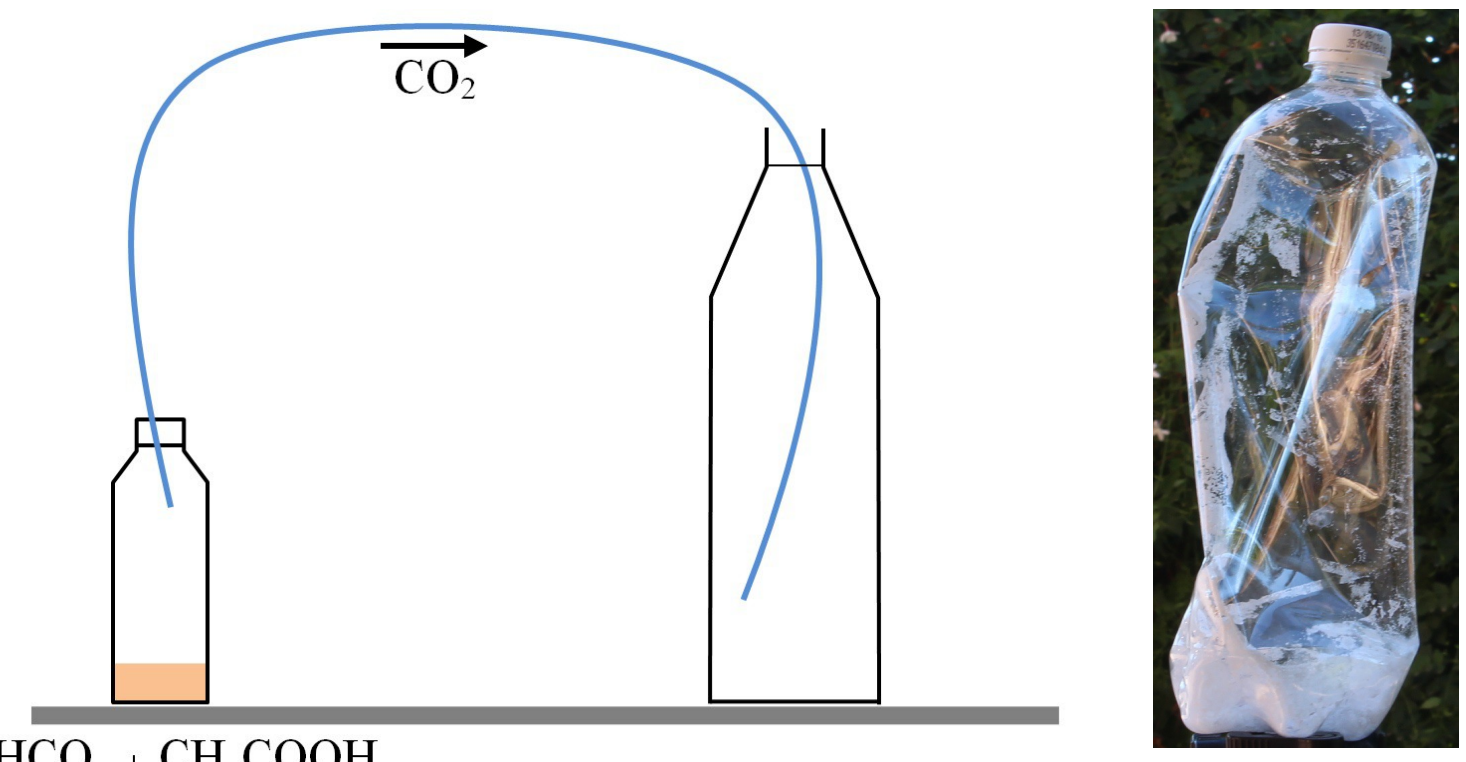

\section{$\mathrm{NaHCO}_{3}+\mathrm{CH}_{3} \mathrm{COOH}$}

Figura 6. (Izda.) El dióxido de carbono se genera en un recipiente auxiliar (puede servir una botella pequeña a la que se le acopla un tubo delgado de plástico) y se trasvasa a la botella a la que, posteriormente, se agregará el hidróxido sódico sólido. Esta segunda botella debe estar abierta inicialmente para que el dióxido de carbono pueda reemplazar al aire que contenía previamente. (Dcha.) Estado final que presenta una botella de plástico tras reaccionar el dióxido de carbono con hidróxido sódico en fase sólida.

\section{Precauciones en el manejo de los reactivos y gestión de residuos}

- El hidróxido sódico puede ocasionar graves quemaduras si entra en contacto con la piel o los ojos. En caso de accidente, deberá añadirse abundante cantidad de agua sobre la zona afectada.

- Por otra parte, es posible que la botella de plástico se fracture como consecuencia de las fuerzas que ejerce la atmósfera sobre ella cuando el dióxido de carbono reacciona con el hidróxido sódico.

- En consecuencia, es imperativo el uso de gafas y guantes de seguridad, así como llevar a cabo todas las operaciones dentro de una cubeta de plástico que actúe como contenedor en el caso de que se produzca algún derrame de las disoluciones. 
- El hidróxido sódico debe conservarse en un recipiente con cierre hermético y con la menor cantidad posible de aire, para minimizar la absorción de la humedad y del dióxido de carbono presentes en el aire.

- Cuando se emplean las cantidades estequiométricas de los tres reactivos necesarios para el experimento, el único residuo que se genera es una disolución básica de carbonato sódico y de acetato de sodio, que se debe gestionar como residuo de baja peligrosidad; de hecho, el carbonato de sodio se ha utilizado durante mucho tiempo como producto de limpieza (sosa para lavandería) para precipitar los iones calcio que disminuyen la eficacia limpiadora de los jabones (Atkins y Jones 1998). Como en la práctica se emplea un exceso de hidróxido sódico (para acelerar su reacción con el dióxido de carbono), la disolución resultante deberá neutralizarse, antes de verterla a la red de alcantarillado, con una disolución de ácido clorhídrico. Esta operación debe ser realizada por una persona experta (Blanco 1983).

\section{Conclusiones}

Este experimento se puede relacionar con contenidos muy diversos: presión y densidad de los gases, fuerzas ejercidas por la atmósfera, neutralización ácido-base, cálculos estequiométricos, conservación de la masa en reacciones químicas y variaciones de energía asociadas a procesos físico-químicos.

Paralelamente, admite diferentes grados de complejidad dependiendo del nivel de conocimientos del alumnado al que se dirija; también podría plantearse como un pequeño trabajo de investigación, entre cuyos objetivos estarían, por ejemplo, el estudio de la variación de la masa del sistema cuando ocurre el aplastamiento por efecto de la presión atmosférica, el diseño de un procedimiento de trasvase del gas de un recipiente a otro, la búsqueda de un método simple para estimar la reducción de la presión que se ha conseguido, etc.

Además, el experimento es sencillo, se lleva a cabo en unos minutos y ofrece unos resultados muy llamativos y fácilmente reproducibles. Si se adoptan las precauciones oportunas, se minimiza el riesgo de manejar hidróxido sódico en pequeñas cantidades. Los reactivos y materiales que se necesitan son fáciles de conseguir y los residuos que se generan son de baja peligrosidad para el medio ambiente.

\section{Referencias}

Atkins P., Jones L. (1998) Quimica, 3ª ed. Barcelona: Omega.

Blanco F. (1983) Manual de laboratorio de Química. Salamanca: librería Cervantes.

Brown T., LeMay H., Bursten B. (1998) Química, la ciencia central, $7^{a}$ ed. México: Prentice Hall.

Heredia-Avalos S. (2006) Experiencias sorprendentes de química con indicadores de $\mathrm{pH}$ caseros. Revista Eureka sobre Enseñanza y Divulgación de las Ciencias 3 (1), 89-103.

IUB (2017) Sodium Hydroxide and Carbon Dioxide: Why it is Important to Keep Your Standard NaOH Solutions Capped. Indiana University Bloomington.

Prolongo-Sarria M. (2013) Trabajos experimentales de química y de física con un estropajo de acero. Anales de Quimica 109 (1), 45-52.

Resnick R., Halliday D., Krane K. (2002) Fisica, $4^{a}$ ed. México: CECSA.

Shakhashiri B. Z. (1985) Chemical demonstrations. A handbook for teachers of chemistry, Vol. 2. Wisconsin: The University of Wisconsin Press. 
Skoog D., West D., Holler F. (1997) Quimica analítica, 6a ed. México: McGrawHill. 